\title{
EU NoE-metamorphose: metamaterials research activities
}

\author{
Ekmel Ozbay
}




\title{
EU NoE metamorphose: metamaterials research activities \\ Ekmel Ozbay* \\ Nanotechnology Research Center, Bilkent University, Bilkent, 06800 Ankara, TURKEY
}

\begin{abstract}
We will present the activities of METAMORPHOSE a network of excellence (NoE) formed under EU-FP6 on the area of metamaterials. The main scientific objective of the partners of this consortium is to develop new types of artificial materials, referred to below as metamaterials, with electromagnetic properties that cannot be found among natural materials. The results of this development should lead to a conceptually new range of radio, microwave, and optical technologies, based on revolutionary new materials made by large-scale assembly of some basic elements (nanoscopic and microscopic) in unprecedented combinations. Further information on this NoE can be found in http://www.metamaterials-eu.org.
\end{abstract}

Keywords: Metamaterial, Negative refraction, negative phase velocity, split ring resonator, left-handed medium,

Metamaterials are artificial electromagnetic (multi-)functional materials engineered to satisfy the prescribed requirements. The prefix meta means after, beyond and also of a higher kind. Superior properties as compared to what can be found in nature are often underlying in the spelling of metamaterial. These new properties emerge due to specific interactions with electromagnetic fields or due to external electrical control.

Electromagnetic metamaterials will play a key role in providing new functionalities and enhancements to the future electronic devices and components, such as high-speed circuits, multifunctional smart miniature antennas and apertures, high-resolution imaging systems, smart skins, and so forth. After all, these and other systems are built on substrates and superstrates whose electromagnetic response functions define the design and performance of the systems. Consider a particular but characteristic example for the applicability of metamaterials: Recently, the theoretical concept of planar perfect lenses with "left-handed" metamaterials was proposed. Such a perfect lens would enable to circumvent resolution limitations in many optical or electromagnetic systems beyond the diffraction limit. Multitudinous applications in many areas of information technology and life science can be envisaged just for this single particular example, like e.g. better imaging systems, higher capacity optical data storage systems, more compact integrated optical telecom solutions, etc. Joint research activities of this Network will include composite materials with extreme electromagnetic properties (such as "left-handed" media and materials with null-valued effective parameters), electrically controllable materials, stop band materials, metageometries like fractals and quasi-periodical structures, artificial surfaces and sheets.

Metamaterials are, in essence, the materials of the future, since the main purpose for their study is to be able to go beyond where naturally occurring substances and current materials research have taken us. By combining different microscopic elements into large-scale designs, one will be able not only to create materials with fundamentally new properties but also to fabricate others that have properties on demand, as required by new technologies. In particular, new electromagnetic properties will allow us to control microwaves, millimetre waves, and optical light in revolutionary ways. This is the context in which the present proposal is framed.

\section{SCIENCE}

While at microwave frequencies the mature printed circuit board technology may be further extended, with possible straightforward transfer to SME's, the development and the realization of metamaterials at optical frequencies requires highly sophisticated technological equipment which can be only found in very well-equipped facilities closely interacting with micro- or nano-electronic and photonics industry. In this frequency range the fabrication of such new artificial materials could require the creation of new molecules or super atoms at the mesoscopic scale and, thus, can involve also chemical and physical laboratories.

Metamaterials, edited by Tomasz Szoplik, Ekmel Özbay, Costas M. Soukoulis, Nikolay I. Zheludev, Proc. of SPIE Vol. 5955, 59550D, (2005) · 0277-786X/05/\$15 · doi: 10.1117/12.621313 
The strategic objective to develop new metamaterials means opening a new branch of research in the multidisciplinary field of material physics, electromagnetics, optics, radio engineering, and electronics. At present, there are no specialists trained in this field. Participating laboratories make high-level but fragmented research and they need coordination and structuring of their efforts.

Integration of theoretical knowledge and modelling capabilities together with fabrication, testing and measurements abilities, as proposed in this application, will allow us to actually develop new materials with predefined electromagnetic properties, which will be then available for use in device applications. This is our main scientific goal, which fully agrees with the NMP priority.

\section{PARTNERS}

The Metamorphose initiative integrates 13 different countries around Europe from North (FIN, S) to South (E, I, GR, $\mathrm{TR}$ ) and from East (PL, RUS) to West (UK, B) passing through its centre (F, D, CH). In all these countries, very advanced technological facilities have been developed (computing centres, nano-electronic facilities, high frequency characterization of materials), all of them being of prime importance in the lasting integration of the Metamorphose Network. To mention a few, the Institute of Electronics, Microelectronics and Nanotechnology in France is one of the largest centres for advanced technology in Europe along with Chalmers and Glasgow nodes, with a well established reputation in Optoelectronics and Nanotechnology. SMARAD and Millilab, two partners in the Finland node are recognized centres for microwave characterization acknowledged as a national Centre of Excellence and by the European Space Agency, whereas the Aachen University has a very high reputation in electro-optic sampling and near field measurements. Spanish and Finnish laboratories were among pioneers in the field of metamaterials. Everyone knows also the high reputation of East-European researchers in the topic of left-handed materials, which was introduced primarily by a Russian researcher, Prof. Veselago.

\section{NETWORK STRUCTURE}

Restructuring and reshaping will be based on a new organizational structure, called Virtual Institute, amalgamating the experience of the researchers involved in the photonic and in the microwave fields on one hand, and on a close interaction between fundamental and device-oriented researchers on the other. This will generate a critical mass of resources and expertise in the emerging area of metamaterial science and technology. Collective management of resources in the restructured research area will avoid costly multidisciplinary environments in each institute involved in the Network by means of providing access to the excellent tools of each of them and perfecting their research and technology skills in that direction, so that only a few institutes will have to acquire the equipment for special fabrication. Following the same approach, we plan to develop a repository of the most advanced simulation tools developed by the partners specialising in the modelling area and make that available to the Network partners. The METAMORPHOSE VI will act as a means of securing long-term, self-sustaining support for European research into metamaterials.

\section{ACTIVITIES}

The Network activities should result in a Common European Research Platform and a Virtual Institute to plan and organize joint research and use and disseminate knowledge. A mature scientific community should be established that would launch Integrated Projects, STREPS, and other joint projects in strategically targeted areas. A program for personnel mobility of researches should be created, and an international $\mathrm{PhD}$ program should be organized, including web-based learning tools and an information platform. In addition, the Virtual Institute should establish and maintain a mechanism for common use of research facilities and manage regular scientific conferences and workshops.

The various activities of the work programme are grouped into three sets, covering each of the main issues of the network activities. The prime role will be played by the program of integrating activities (JPI) whose main output will be restructured European research platform in the field of metamaterials. The program for jointly executed research activities (JPR) will set up new joint research projects. Finally, the activities to spread excellence (JPS) contain tasks related to spreading excellence and dissemination of research results towards the scientific community and the industry. 


\section{INTEGRATING ACTIVITIES (JPI)}

The main long-term goal of the integration workpackages will be the creation of a Virtual Institute where all the metamaterials related joint research activities carried out within the partner institutions will be integrated and coordinated by one organization.

\section{JOINTLY EXECUTED RESEARCH ACTIVITIES (JPR)}

The Network goals will require the collaboration of several groups that will share their knowledge and facilities working on new joint research projects. Workpackage 2.1 "Coordinated programming and adaptation of research activities. Definition of future needs and specifications" will propose new research projects to be approved and launched. This work will be done based on the review and assessment of technical areas.

\section{SPREADING OF EXCELLENCE (JPS)}

The proposed new series of conferences on metamaterials will agglutinate around the core groups in this network the available knowledge in this field, and at the same time it will contribute to dissemination of the outcoming results among a broad scientific community. The NoE will provide financial support to organise these conferences. Furthermore, a database will be created that will describe the main findings in metamaterials as they are generated. Our purpose is that it would become a central international reference in this field, where the scientific community at large can benefit from our knowledge, and where SMEs can find a suitable reference that contributes to spread it towards more specific applications. Finally, courses (a specialized $\mathrm{PhD}$ program) organised within the network will be the key ingredient to cross the bridge between out cutting edge activities in research and text books where metamaterials become an important tool in the curricula of materials scientists and engineers.

The NoE is a suitable instrument to organize research, provide the financial support for personnel mobility and facility sharing required to integrate the proposed activities. In particular:

Horizontal integration. A fluid communication to transfer knowledge along the route from the theory through modelling and testing to applications. Exchanges between groups that specialize at different stages of this process will be needed to achieve such fluid communication.

Vertical integration. A platform that will be created to serve metamaterials by providing knowledge about optics, microwaves, nano-science and solid-state physics needs also funding for exchanges and also to host meetings where the integrated activities and their specific location in this platform will be worked out in more detail.

This effort will have a dramatic impact on some emerging technologies, in particular communications technologies that totally depend on the propagation and processing of electromagnetic signals. The promising field of metamaterials is relatively unexploited and there are many companies and research groups worldwide that are orienting their efforts towards solving technological problems relying on them. An European lead over the already existing American effort on this front is therefore an excellent bet towards competitiveness and sustainable development in new technologies, and in particular in communication applications, where this proposal can play a substantial role by contributing to generate new materials and to discover exciting new phenomena that will solve long standing problems while opening new opportunities.

We are pursuing breakthroughs in the form of novel properties and phenomena of engineered materials, which we expect will revolutionize the field of communications technologies. While some of this research will find nearly immediate applications, this is nonetheless a calculated risk in the long-term, since the metamaterials that will be made available within this network will undoubtedly increase the choices for new research and technologies in the future, while the scientific knowledge that will be produced will contribute to original perspective views in the understanding of materials research. Therefore, the long-term repercussion of this work can be extraordinary. The NoE will also support high-risk activities to design and develop new structures with defined characteristics leading to new industrial 
applications. Computational strategies, numerical simulations, theoretical modelling, and experimental investigations will be the key tools.

\section{CONTRIBUTION TO GENERATE KNOWLEDGE}

The fundamental idea behind the research in metamaterials is to utilise already existing knowledge about the behaviour of basic materials as building blocks of more complex structures. This provides a perfect opportunity to promote real breakthroughs in understanding materials phenomena, as many unexpected properties of this general category of materials remain to be found. Good examples of this have been recently reported (e.g., superlenses using newly designed left-handed materials or laser collimation by means of materials with vanishing index of refraction).

New ways to design metamaterials, to fabricate them, to analyse and to integrate them in different technological contexts will be the main contribution to the generation of knowledge from this network. Indeed, there is an urgent need to produce this savoir faire that will make metamaterials a standard solution in future and emerging technologies. Metamaterials are certainly there, waiting to be investigated, and this network will provide the necessary framework for integration of the key elements that will make this possible to EU's advantage. In particular, applications for radiation over different wavelength scales ranging from the microwaves to the optical regime is very suited to designing new materials with electromagnetic properties on demand with important technological areas of specific application.

\section{POTENTIAL IMPACT AND APPLICATION}

The application of the results of this project will increase the quality of life of the European citizens. Perhaps the most immediate applications of new metamaterials are foreseen in the area of telecommunications and information processing. One of the primary objectives of the EU is to bring the Information Society near to ALL. In order to achieve this goal, the current "local loop" accesses have to be overcome by means of wireless communication systems as it is specified in the eEurope impact and priorities document. These systems must increase their operational frequency spectrum (around $40 \mathrm{GHz}$ according to the decision of the European Radio Conference of 1.6.99) to create broadband high-speed infrastructures. These allow any user to connect to the emerging high bitrate Internet services, multimedia wireless systems and digital television. Every citizen will be equipped with tools and skills needed to live and work in the new Information Society. New devices, components, and systems need to be developed to underpin new infrastructure of the Information Society. In addition, new technologies have to be promoted to strengthen the European leadership in the mobile communications market.

From these premises, METAMORPHOSE addresses the fundamental societal needs by providing novel metamaterial technologies which lay the foundation for advances in development of consumer and military applications for the new Information Society infrastructure. Novel components and devices for these emerging systems will be explored, designed, fabricated, and measured. Furthermore, properties of metamaterials in the sub-millimetre wave frequency range will be studied to anticipate future applications, making Europe a pioneer in these technologies. These frequency ranges present enormous potential for ultra high band communications, CCD-imaging and sensing for biomedical applications, environmental monitoring, and food quality control.

Progress in metamaterial research will trigger advances in other priority action lines of the EU such as e-health and eresearch. For example, the use of metamaterials in medical instrumentation will enhance functionality of existing equipment that will have positive impact on the quality of health services. This like other major technological advances will be the enabling mechanisms for improving quality of life and prosperity of the EC. In the European scientific community it will mean a technological edge for European laboratories. This Network will have a direct impact on Europe at grass-roots level which will begin to take effect in the long term.

Besides telecommunications, other applications in which metamaterial technology can be directly useful are Medical, Security, Chemical, and Aeronautics. To give a few examples, let us concentrate on the sub-millimetre frequency range devices, where the metamaterial technology is expected to find immediate applications. We can mention that in the medical, dermatology and cosmetic sectors, sub-millimetre wave imaging is rapidly becoming recognised as a totally 
new diagnostic technique. By observing these types of waves, it is possible to see through many optically opaque materials. Sub-millimetre waves could provide an image that has X-ray-like properties without the use of potentially harmful radiation. As sub-millimetre waves can penetrate the uppermost layers of skin, they may provide the missing quick diagnostic tool for skin cancer. Other possibilities include looking behind a dressing to see if a wound is healing correctly. An application of a different nature could be the detection of chemical and biological threats. As all materials emit sub-millimetre waves, each having its own frequency pattern as a kind of 'fingerprint', it could be possible to identify not only the existence of harmful substances (e.g. powder in envelopes and postal parcels), but also which kind of material is enclosed. In airports, it could be possible to see through clothes and identify weapons, without resorting to the metal-detection techniques used today. Even non-metal explosives could be spotted since they may have their own sub-millimetre 'fingerprints'.

It is clear that the integration of the new metamaterial technology components in the broadband communication market will promote the creation of new employment opportunities all over Europe. This will happen directly by the professionals dedicated to the design and manufacturing of the systems themselves and, most importantly, indirectly by creating spin-offs in the areas of new material technology through supporting new Internet software applications, digital $\mathrm{TV}$, multimedia services, etc. It should be stressed that, despite the large potential of metamaterial systems in the microwave and sub-millimetre frequency ranges, there is a lack of European human potential with the necessary interdisciplinary qualifications. Therefore, METAMORPHOSE will also contribute to provide a solid base for the involved researchers who will transfer their knowledge in this developing research field to the scientific community. A team encompassing competent multidisciplinary partners is the only way to develop now the human potential required to spear ahead in this rapidly emerging field. The impact will be direct and come into effect in a medium/long term.

Finally, the effect on preserving or enhancing the environment is obviously indirect but its impact will nonetheless be real. The application of these novel metamaterials in the development of components and sub-systems at the more challenging millimetre and sub-millimetre wave frequency range offers an attractive application in the area of environmental monitoring. The interest in environmental monitoring comes from the fact that there are several very important processes taking place in the atmosphere that deserve our attention, like the greenhouse effect and ozone depletion. There is an ever-growing awareness on the possible detrimental effects of man's activities on the climate. Current high levels of chlorine and bromine loading in the stratosphere are expected to peak early in the next decade and it is extremely important to quantify the anticipated changes in ozone and temperature by making the appropriate quantitative observations. Sub-millimetre wave frequencies can be used to obtain information on chlorine chemistry in the stratosphere, providing data for studies on ozone depletion mechanisms, while millimetre wave frequencies can focus on exchanges between troposphere and stratosphere, bringing complementary information useful for studies on global changes. In remote sensing, metamaterials should allow us to fabricate low-cost and low-weight ground-based and satellite-based antennas with unprecedented capabilities in terms of resolution and frequency coverage.

*ozbay@fen.bilkent.edu.tr 